\title{
Significance of Rarefaction, Streamwise Conduction, and Viscous Dissipation on the Extended Graetz-Nusselt Problem: The Case of Finite-Length Microchannels with Prescribed Wall Heat Flux
}

\author{
Antonio Brasiello $\mathbb{i D}^{1,2}$ and Alessandra Adrover $\mathbb{i D}^{1}$ \\ ${ }^{1}$ Dipartimento di Ingegneria Chimica, Materiali e Ambiente, Sapienza Università degli Studi di Roma, Via Eudossiana 18, \\ Roma 00184, Italy \\ ${ }^{2}$ INSTM Consorzio Interuniversitario Nazionale per la Scienza e Tecnologia dei Materiali, Via G. Giusti 9, Firenze 50121, Italy
}

Correspondence should be addressed to Antonio Brasiello; antonio.brasiello@uniroma1.it

Received 28 March 2020; Accepted 28 July 2020; Published 25 August 2020

Academic Editor: Jitendra Kumar

Copyright (c) 2020 Antonio Brasiello and Alessandra Adrover. This is an open access article distributed under the Creative Commons Attribution License, which permits unrestricted use, distribution, and reproduction in any medium, provided the original work is properly cited.

\begin{abstract}
The article addresses the extended Graetz-Nusselt problem in finite-length microchannels for prescribed wall heat flux boundary conditions, including the effects of rarefaction, streamwise conduction, and viscous dissipation. The analytical solution proposed, valid for low-intermediate Peclet values, takes into account the presence of the thermal development region. The influence of all transport parameters (Peclet Pe, Knudsen Kn, and Brinkman Br) and geometrical parameters (entry length and microchannel aspect ratio) is investigated. Performances of different wall heat flux functions have been analyzed in terms of the averaged Nusselt number. In the absence of viscous dissipation $\mathrm{Br}=0$, the best heating protocol is a decreasing wall heat flux function. In the presence of dissipation $\mathrm{Br}>0$, the best heating protocol is a uniform wall heat flux.
\end{abstract}

\section{Introduction}

A correct estimation of heat and mass transfer coefficients is a powerful tool in the design of heat exchangers [1], mass transfer equipment and reactors [2], and microdevices [3, 4] for chemical [5], biomedical [6,7], and pharmaceutical applications $[8,9]$.

Focusing on laminar forced convection of an incompressible fluid in a duct, the estimation of transport coefficients requires the solution of the classical Graetz-Nusselt problem $[2,10]$. Originally proposed for a sudden step change of the wall temperature at some positions along the duct and no axial diffusion, the Graetz-Nusselt problem is valid for both heat and mass transfer. It has been solved in transient and steady-state [11], for Dirichlet and Neumann boundary conditions [12], for different wall shape and curvature $[9,13,14]$, for non-Newtonian fluids [15], and for counterflow streams [16], in the presence of high viscous dissipation [17], axial diffusion $[18,19]$, and simultaneous heat and mass transfer $[20,21]$.

As the size of the channel is reduced, the no-slip boundary condition needs to be modified because velocity slippage [22-25] and temperature jump may occur at the wall. The so-called "extended" Graetz problem in microtubes, accounting for rarefaction and viscous dissipation, has been recently investigated by Cetin et al. $[26,27]$ and by Jeong and Jeong $[28,29]$ by means of eigenfunction expansion (including streamwise conduction) and by Tunc and Bayazitoglu [30] using an integral transform technique (neglecting streamwise conduction).

In all the above-mentioned papers, it is assumed that the fluid enters the semi-infinite microchannel $(z \geq 0)$ as a fully developed isothermal flow, that is, $T=T_{0}$ at $z=0$. However, this boundary condition at $z=0$ may be extremely restrictive, especially in the case of laminar conditions and low Peclet values [31]. If axial conduction is important, then a sizeable amount of heat is conducted upstream the entrance 
cross section $z=0$ into the hydrodynamic development region $z<0$. Therefore, a temperature distribution is built up for $z<0$ and this may significantly affect the temperature downstream. For this reason, Barletta and Magyari [32] addressed the problem of the thermal entrance forced convection in a circular duct with a prescribed wall heat flux distribution, including the effect of viscous dissipation but neglecting heat axial conduction. The presence of an upstream insulated region in microchannels has been also accounted for also by Knupp et al. [33-35] by combining the Generalized Integral Transform Technique (GITT) and a single domain reformulation strategy, aimed at providing hybrid numerical/analytical solutions to convection/diffusion problems. No viscous dissipation effects or slip boundary conditions are accounted for while wall conjugation effects are taken into account.

The present paper presents an analytical solution of the extended Graetz problem in finite-length microtubes including the effects of rarefaction, streamwise conduction, and viscous dissipation. The solution, taking into account the presence of the thermal development region, is valid for low-intermediate Peclet values Pe and for the prescribed heat flux boundary conditions (no wall conjugation effects).

In dealing with finite-length channels, in the presence of axial dispersion and wall heat flux, one issue to be addressed is the proper boundary conditions at the inlet and outlet sections. For this reason, in the problem formulation, three distinct regions along the microchannel have been considered (see Figure 1): a thermally insulated region (length $L^{-}$, wall heat flux $Q_{w}=0$ ), followed by a heat transfer region with length $L$ and prescribed wall heat flux $Q_{w}(z)$, followed by a third thermally insulated region (length $L^{+}$, wall heat flux $Q_{w}=0$ ).

The analytical solution is compared with the numerical results obtained by means of the finite elements method (FEM Comsol 3.5) in a wide range of Pe and for different wall heat flux profiles. The range of validity of the analytical solution is investigated in detail.

From the temperature field, the local Nusselt axial profile and the average Nusselt number are obtained as a function of the transport parameters, that is, the Peclet number Pe, the Knudsen number $\mathrm{Kn}$, and the Brinkman number $\mathrm{Br}$. The influence of geometrical parameters, that is, the aspect ratio diameter-over-length $D / L$ and the length of the upstream section $L^{-} / L$, is also addressed.

The solution, valid for small-intermediate values of $\mathrm{Pe}$, is presented for cylindrical and flat rectangular microchannels (see Appendix A). A list of symbols is reported in Appendix B.

\section{Statement of the Problem and Analytical Solution}

Let us consider a Newtonian fluid with constant thermodynamic properties entering, at $z=-L^{-}$, into a finite-length microtube of radius $R$, diameter $D$, and total axial length $\left(L^{+}+L+L^{-}\right)$, that is, $L^{-} \leq z \leq L+L^{+} .\left(\pi R^{2}\right)\left(\rho_{f} c_{p} U\right) T_{0}$ is the inlet convective flux at $z=L^{-}, U$ being the average cross section velocity. The microtube is thermally insulated for $L^{-} \leq z<0$ and for $L<z \leq L+L^{+}$. Let us indicate with $Q_{w}(z)$ the prescribed wall heat flux for the heat transfer region $0 \leq z \leq L$ and with $Q_{\mathrm{av}}=(1 / L) \int_{0}^{L} Q_{w}(z) \mathrm{d} z \neq 0$ its average value.

Let $V_{z}(r)$ be the fully developed first-order velocity profile in slip-flow regime. In terms of the dimensionless radial coordinate, $\rho=r / R, V_{z}(r)$ attains the form

$$
\begin{aligned}
V_{z}(\rho) & =2 U \frac{1-\rho^{2}+4(2-F / F) K n}{1+8((2-F) / F) K n}=2 U \frac{1-\rho^{2}+4 \mathrm{Kn}}{1+8 \mathrm{Kn}} \\
& =U\left(2\left(1-\rho^{2}\right) \chi+1-\chi\right), \\
\chi & =\frac{1}{1+8 \mathrm{Kn}}, \\
\mathrm{Kn} & =\frac{\lambda}{D},
\end{aligned}
$$

where $\mathrm{Kn}$ is the Knudsen number.

A first-order model for rarefaction effects is adopted, being valid for $0.001 \leq \mathrm{Kn} \leq 0.1$ [36] with a tangential momentum accommodation coefficient $F=1$ [30]. The velocity profile reduces to the classical no-slip parabolic velocity profile for $\mathrm{Kn}=0$.

By introducing the dimensionless space variables $\rho=r / R$, $\zeta=z / L$, temperature $\theta=\left(T-T_{0}\right) k /\left(Q_{\mathrm{av}} R\right)$, and velocity $v(\rho)=V_{z} / U$, the starting point of the subsequent analysis is the steady state heat-balance equation, accounting for radial conduction, axial conduction, and convection and viscous dissipation:

$$
\begin{aligned}
& -\frac{\operatorname{Pe} \alpha}{4} v(\rho) \frac{\partial \theta}{\partial \zeta}+\frac{1}{\rho} \frac{\partial}{\partial \rho}\left(\rho \frac{\partial \theta}{\partial \rho}\right)+\frac{\alpha^{2}}{4} \frac{\partial^{2} \theta}{\partial \zeta^{2}}+32 \mathrm{Br} \chi^{2} \rho^{2}=0, \\
& -\beta^{-}<z<1+\beta^{+},
\end{aligned}
$$

where the Peclet number Pe and the Brinkman number $\mathrm{Br}$ appear as

$$
\begin{aligned}
& \mathrm{Pe}=\frac{U D}{k / \rho_{f} c_{p}}, \\
& \mathrm{Br}=\frac{\mu U^{2}}{Q_{\mathrm{av}} D},
\end{aligned}
$$

together with the geometric dimensionless parameters $\alpha, \beta^{+}$, and $\beta^{-}$

$$
\begin{gathered}
\alpha=\frac{D}{L}, \\
\beta^{-}=\frac{L^{-}}{L}, \\
\beta^{+}=\frac{L^{+}}{L} .
\end{gathered}
$$

By further introducing the dimensionless axial convective flux

$$
J_{c}(\rho, \zeta)=\frac{\operatorname{Pe} \alpha}{4} v(\rho) \theta(\rho, \zeta),
$$

the following boundary conditions apply 


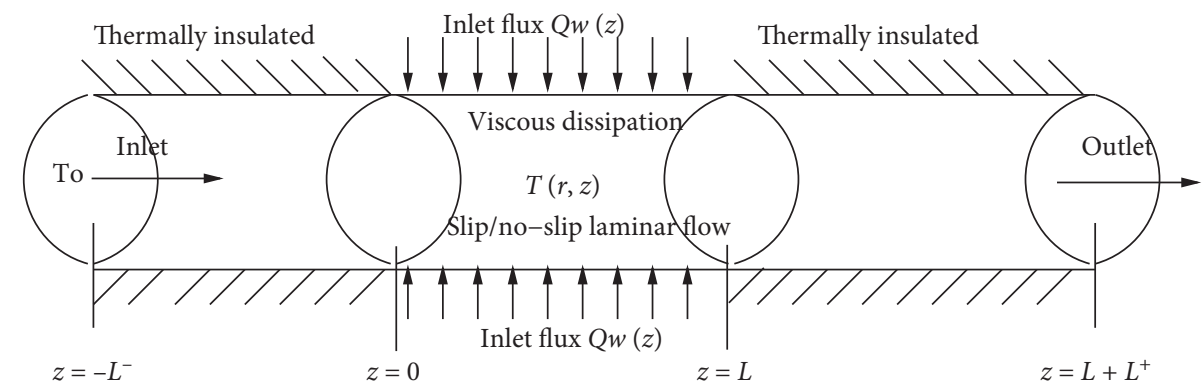

FIgURE 1: Schematic representation of the physical domain and boundary conditions.

$$
\begin{gathered}
\left.J_{c}\right|_{\zeta=-\beta^{-}}=0, \\
\frac{\partial}{\partial \zeta} \int_{0}^{1} J_{c} 2 \pi \rho d \rho=16 \pi \mathrm{Br} \chi^{2}, \quad \text { at } \zeta=1+\beta^{+}, \\
\left.\frac{\partial \theta}{\partial \rho}\right|_{\rho=0}=0, \\
\left.\frac{\partial \theta}{\partial \rho}\right|_{\rho=1}=F_{w}(\zeta), \\
F_{w}(\zeta)= \begin{cases}0, & \text { for }-\beta^{-} \leq \zeta<0, \\
q_{w}(\zeta)=\left(Q_{w}(\zeta) / Q_{\mathrm{av}}\right), & \text { for } 0 \leq \zeta \leq 1, \\
0, & \text { for } 1<\zeta \leq 1+\beta^{+} .\end{cases}
\end{gathered}
$$

The outlet boundary condition equation (6) at $\zeta=1+$ $\beta^{+}$is an integral version of the Danckwerts outlet boundary condition, usually adopted for finite-length channels, and implies zero conductive axial heat flux at the outlet section. Its integral version [37] (integral over the radial cross-section) allows us to take into account the heat generated by viscous dissipation and is in agreement with the asymptotic condition usually adopted for infinitely long channels.

2.1. Analytical Solution. At low-intermediate values of the Peclet number, the temperature profile exhibits a weak dependence on the radial coordinate $\rho$ so that it can reasonably assumed that the dimensionless temperature can be written as the linear combination of two functions:

$$
\begin{aligned}
& \theta(\rho, \zeta)=\theta_{b}(\zeta)+\phi(\rho, \zeta), \\
& \theta_{b}(\zeta)=\frac{1}{\pi} \int_{0}^{1} \theta v(\rho) 2 \pi \rho \mathrm{d} \rho
\end{aligned}
$$

where $\theta_{b}(\zeta)$ is the dimensionless bulk temperature (mixing cup temperature) and $\phi(\rho, \zeta)$ is an auxiliary function satisfying the integral constrain:

$$
\frac{1}{\pi} \int_{0}^{1} \phi(\rho, \zeta) v(\rho) 2 \pi \rho \mathrm{d} \rho=0 .
$$

The auxiliary function $\phi(\rho, \zeta)$ accounts for the temperature dependence on the radial coordinate, that is, $(\partial \theta / \partial \rho)=(\partial \phi / \partial \rho)$, and is a slowly varying function of the axial coordinate $\zeta$, so that

$$
\begin{gathered}
\frac{\partial \phi}{\partial \zeta} \ll \frac{\mathrm{d} \theta_{b}}{\mathrm{~d} \zeta}, \\
\frac{\partial^{2} \phi}{\partial \zeta^{2}} \ll \frac{\mathrm{d}^{2} \theta_{b}}{\mathrm{~d} \zeta^{2}} .
\end{gathered}
$$

By substituting (9) into the balance equation (2) and into the boundary conditions equations (6)-(8) and making use of the simplifying assumption equation (11), one obtains

$$
\begin{aligned}
\frac{\partial \phi}{\partial \zeta} & \ll \frac{\mathrm{d} \theta_{b}}{\mathrm{~d} \zeta}, \\
\frac{\partial^{2} \phi}{\partial \zeta^{2}} & \ll \frac{\mathrm{d}^{2} \theta_{b}}{\mathrm{~d} \zeta^{2}}, \\
\left.\theta_{b}\right|_{\zeta=-\beta} & =0, \\
\frac{\operatorname{Pe} \alpha}{4} \frac{\mathrm{d} \theta_{b}}{\left.\mathrm{~d} \zeta\right|_{\zeta=1+\beta^{+}}} & =16 \mathrm{Br} \chi^{2}, \\
\left.\frac{\partial \phi}{\partial \rho}\right|_{\rho=0} & =0, \\
\left.\frac{\partial \phi}{\partial \rho}\right|_{\rho=1} & =F_{w}(\zeta) .
\end{aligned}
$$

In (12), both $\theta_{b}$ and $\phi$ still appear. However, an independent equation for the bulk temperature $\theta_{b}(\zeta)$ can be obtained as follows. By integrating the balance equation (2) over the entire radial cross section, one obtains

$$
-\frac{\operatorname{Pe} \alpha}{4} \frac{\mathrm{d} \theta_{b}}{\mathrm{~d} \zeta}+\frac{\alpha^{2}}{4}\left(\frac{\mathrm{d}^{2} \theta_{b}}{\mathrm{~d} \zeta^{2}}+\frac{\partial^{2} \phi}{\partial \zeta^{2}}\right)+16 \mathrm{Br} \chi^{2}+2 F_{w}(\zeta)=0
$$

By further enforcing solely the second-order simplifying assumption $\left(\partial^{2} \phi / \partial \zeta^{2}\right) \ll\left(\mathrm{d}^{2} \theta_{b} / \mathrm{d} \zeta^{2}\right)$, one arrives at the following equation for the dimensionless bulk temperature $\theta_{b}(\zeta)$ 


$$
\begin{aligned}
-\frac{\operatorname{Pe} \alpha}{4} \frac{\mathrm{d} \theta_{b}}{\mathrm{~d} \zeta}+\frac{\alpha^{2}}{4} \frac{\mathrm{d}^{2} \theta_{b}}{\mathrm{~d} \zeta^{2}}+16 \operatorname{Br} \chi^{2}+2 F_{w}(\zeta) & =0, \\
\theta_{b}(-\beta) & =0, \\
\left.\frac{\operatorname{Pe} \alpha}{4} \frac{\mathrm{d} \theta_{b}}{\mathrm{~d} \zeta}\right|_{\zeta=1+\beta^{+}} & =16 \mathrm{Br} \chi^{2} .
\end{aligned}
$$

Equations (16) and (17) can be analytically solved, thus obtaining

$$
\begin{aligned}
\theta_{b}(\zeta), \\
g(\zeta<0)=\frac{P e}{\alpha} e^{P e \zeta / \alpha} \int_{0}^{1} e^{-P e \zeta_{1} / \alpha} q_{w}\left(\zeta^{\prime}\right) \mathrm{d} \zeta^{\prime}, \\
g(0 \leq \zeta \leq 1)=\frac{P e}{\alpha} e^{P e \zeta / \alpha} \int_{\zeta}^{1} e^{-P e \zeta^{\prime} / \alpha} q_{w}\left(\zeta^{\prime}\right) \mathrm{d} \zeta^{\prime}, \\
g(\zeta>1)=0, \\
I F_{w}(\zeta<0)=0, \\
I F_{w}(\zeta>1)=1, \\
I F_{w}(0 \leq \zeta \leq 1)=\int_{0}^{\zeta} q_{w}\left(\zeta^{\prime}\right) \mathrm{d} \zeta^{\prime} .
\end{aligned}
$$

It can be observed that, by neglecting the viscous dissipation, that is, by setting $\mathrm{Br}=0$, the bulk temperature profile is independent of the velocity profile, resulting in the same for slip $(K n \neq 0, \chi \neq 1)$ and for no-slip flows $(\mathrm{Kn}=0, \chi=1)$.
By substituting the expression for $\theta_{b}$, (18) into the transport equation (12), one arrives at the following equation for the auxiliary function $\phi(\rho, \zeta)$ :

$$
\begin{aligned}
\frac{1}{\rho} \frac{\partial}{\partial \rho}\left(\rho \frac{\partial \phi}{\partial \rho}\right)= & 2\left(g(\zeta)+8 B r \chi^{2}\right)(v(\rho)-1) \\
& +2\left(F_{w}(\zeta)+8 B r \chi^{2}\right)-32 \mathrm{Br} \chi^{2} \rho^{2}
\end{aligned}
$$

By solving (24) along the radial coordinate, one arrives at the following expression for $\phi(\rho, \zeta)$ satisfying the boundary conditions equation (14):

$$
\begin{aligned}
\phi(\rho, \zeta)= & {\left[F_{w}(\zeta)+8 \operatorname{Br} \chi^{2}(1+\chi)+g(\zeta) \chi\right] \frac{\rho^{2}}{2}+} \\
& -\left[8 \operatorname{Br} \chi^{2}(1+\chi)+g(\zeta) \chi\right] \frac{\rho^{4}}{4}+C(\zeta) .
\end{aligned}
$$

The function $C(\zeta)$ can be determined by enforcing the integral constraint equation (10):

$$
\begin{aligned}
C(\zeta)= & g(\zeta) \chi\left(\frac{\chi}{24}-\frac{1}{6}\right)+F_{w}(\zeta)\left(\frac{\chi}{12}-\frac{1}{4}\right) \\
& +\operatorname{Br} \chi^{2}\left(\frac{\chi^{2}}{3}-\chi-\frac{4}{3}\right)
\end{aligned}
$$

It can be observed that, in the case of very long-thin channels, that is, for $\alpha \longrightarrow 0$, the analytical solution, equations (18)-(23),(25)-(26), strongly simplifies because

$$
\lim _{\mathrm{Pe} / \alpha \longrightarrow \infty} g\left(\zeta, \frac{\mathrm{Pe}}{\alpha}\right)=F_{w}(\zeta)
$$

and the dimensionless temperature profile attains the form

$$
\begin{aligned}
\theta_{\infty}(\rho, \zeta)= & \underbrace{\frac{8}{\operatorname{Pe}^{2}}\left[F_{w}(\zeta)+\frac{\operatorname{Pe}}{\alpha}\left(I F_{w}(\zeta)+8 \operatorname{Br} \chi^{2}\left(\left(\zeta+\beta^{-}\right)\right)\right]\right.}_{\theta_{b}(\zeta)}+ \\
& +\underbrace{(1+\chi)\left[F_{w}(\zeta)+8 B r \chi^{2}\right] \frac{\rho^{2}}{2}-\left[8 \operatorname{Br} \chi^{2}(1+\chi)+F_{w}(\zeta) \chi\right] \frac{\rho^{4}}{4}}_{\phi(\rho, \zeta)}+ \\
& +\underbrace{F_{w}(\zeta)\left(-\frac{1}{4}-\frac{\chi}{12}+\frac{\chi^{2}}{24}\right)+\operatorname{Br} \chi^{2}\left(-\frac{4}{3}-\chi+\frac{\chi^{2}}{3}\right)}_{C(\zeta)},
\end{aligned}
$$

representing the asymptotic (infinite length of the heating section) fully developed temperature profile.

For $q_{w}(\zeta)=1$ (i.e., constant wall heat flux) and for $\beta^{-}=$ 0 (i.e., no upstream section) one recovers the fully developed temperature profile $\theta_{\infty}$ for a constant wall heat flux $[26,27]$, usually written in terms of the dimensionless axial coordinate $\xi=z /(P e R)$ :

$$
\begin{aligned}
\theta_{\infty}(\rho, \zeta)= & 4\left[1+8 \mathrm{~B} r \chi^{2}\right] \xi+\left[1+8 \mathrm{Br} \chi^{2}\right](1+\chi) \frac{\rho^{2}}{2}+ \\
& -\left[8 \mathrm{Br} \chi^{2}(1+\chi)+\chi\right] \frac{\rho^{4}}{4}+\frac{8}{\mathrm{Pe}^{2}}+ \\
& +\left(-\frac{1}{4}-\frac{\chi}{12}+\frac{\chi^{2}}{24}\right)+\operatorname{Br} \chi^{2}\left(-\frac{4}{3}-\chi+\frac{\chi^{2}}{3}\right)
\end{aligned}
$$




\section{Comparison with Numerical Results}

In order to verify the correctness of the analytical solution equations (18)-(23),(25)-(26) and to identify the limits of validity in terms of $\mathrm{Pe}$ and $\alpha$, the transport problem equations (2)-(8) have been solved with finite elements method (FEM, Comsol 3.5 a).

The convection-diffusion package in stationary conditions has been used. Lagrangian quadratic elements are chosen. The linear solver adopted is UMFPACK, with relative tolerance $10^{-12}$.

The number of finite elements is $3 \times 10^{5}$ with a nonuniform mesh. The maximum element size in all the three subdomains $(\zeta<0,0 \leq \zeta \leq 1$, and $\zeta>1)$ is $1 \times 10^{-2}$. Smaller elements have been located close to the boundaries. Specifically, a maximum element size of $2 \times 10^{-4}$ is chosen at the external boundary $(0 \leq \zeta \leq 1, \rho=1)$ and at the internal cross sections $(\zeta=0,1,0 \leq \rho \leq 1)$, that is, at the internal boundaries between the insulated and the heated regions.

Figure 2 shows the computational domain and the mesh element size adopted for the entire set of simulations with $\beta^{-}=\beta^{+}=1$. The mesh adopted guarantees an accurate description of temperature gradients for all the wall heat flux functions adopted (continuous or discontinuous at $\zeta=0,1$ ) and for all the values of Pe analyzed.

As test cases, three different expressions for the wall heat flux function $q_{w}(\zeta)$ have been considered.

(1) A triangular function:

$$
q_{w}(\zeta)= \begin{cases}4 \zeta, & \text { for } \leq \zeta<1 / 2, \\ q_{w}(\zeta)=4-4 \zeta, & \text { for } 1 / 2 \leq \zeta \leq 1\end{cases}
$$

for which the corresponding integral functions $g(\zeta)$ and $I F_{w}(\zeta)$ can be easily computed (nonreported here for the sake of brevity).

(2) An exponential function [19], decreasing along $\zeta$ for $n>0$ and increasing along $\zeta$ for $n<0$ :

$$
q_{w}(\zeta)=\frac{n e^{-n \zeta}}{1-e^{-n}}
$$

The corresponding integral functions $g(\zeta)$ and $I F_{w}(\zeta)$ attain the form

$$
\begin{aligned}
g(\zeta<0) & =\frac{n e^{\mathrm{Pe} \zeta / \alpha}}{1-e^{-n}}\left(\frac{\mathrm{Pe} / \alpha}{(\mathrm{Pe} / \alpha)+n}\right)\left[1-e^{-((\mathrm{Pe} / \alpha)+n)}\right], \\
g(\zeta>1) & =0 \\
g(0 \leq \zeta \leq 1) & =\frac{n e^{-n \zeta}}{1-e^{-n}}\left(\frac{\mathrm{Pe} / \alpha}{(\mathrm{Pe} / \alpha)+n}\right)\left[1-e^{-((\mathrm{Pe} / \alpha)+n)(1-\zeta)}\right], \\
I F_{w}(\zeta<0) & =0 \\
I F_{w}(\zeta>1) & =1 \\
I F_{w}(0 \leq \zeta \leq 1) & =\frac{1-e^{-n \zeta}}{1-e^{-n}} .
\end{aligned}
$$

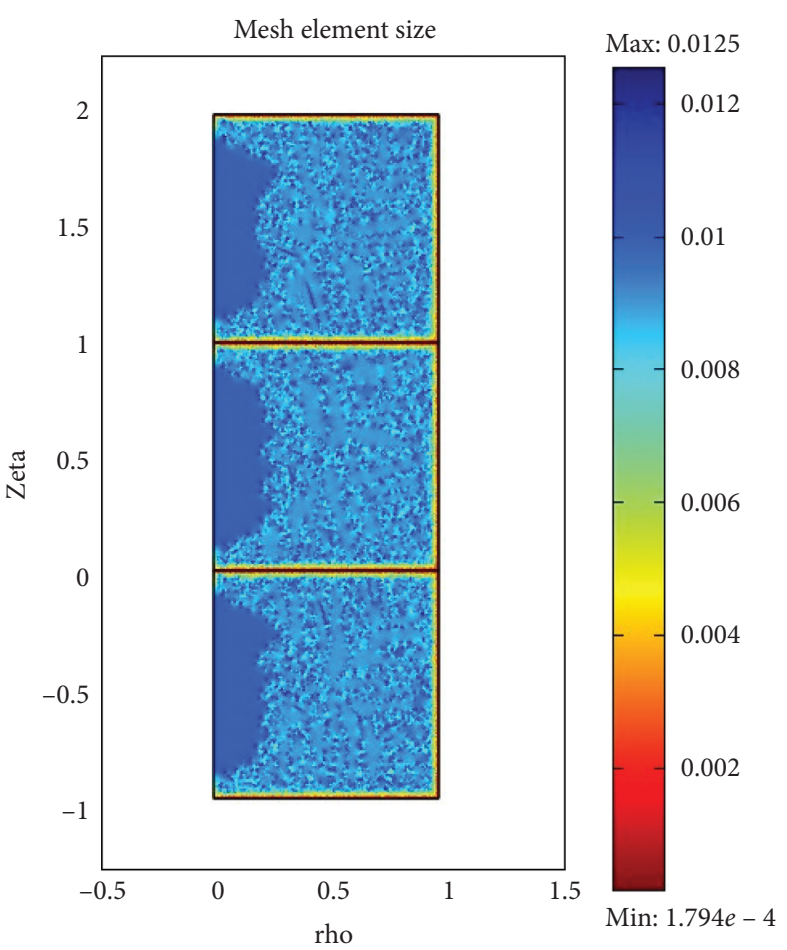

FIGURE 2: Color plot of the mesh element size [dimensionless units] for the computational domain $\left(-\beta^{-} \leq \zeta \leq 1+\beta^{+}, 0 \leq \rho \leq 1\right)$ for $\beta^{-}=\beta^{+}=1$.

(3) The uniform wall heat flux function $q_{w}(\zeta)=1$ that can be obtained as a limiting case of the exponential function for $n \longrightarrow 0$.

3.1. Temperature Profiles. Figure 3 shows the excellent agreement between numerical and analytical results for the temperature profiles in both the upstream and downstream sections for the triangular wall heat flux function. In this case, the function $F_{w}(\zeta)$ is continuous at $\zeta=0$ and $\zeta=1$ so that the simplifying assumption equation (11)fd11 works well in the entire transport domain. The results are shown for a high $\mathrm{Pe}$ value $\mathrm{Pe}=100$ at the limit of validity of the assumption equation (9)fd9.

It can be observed that there is a significant effect of the upstream thermally insulated section because the bulk temperature is order of unity, for $\mathrm{Br}=0.1$, when the fluid enters the heated section. Even in the case of low dissipation, that is, $\mathrm{Br}=0.01$, the temperature profile is significantly affected by the presence of the upstream section. The behaviour in the thermal development region (close to $\zeta=0$ ) is well described by the analytical solution.

Figures 4(a)and 4(b) show the comparison between analytical and numerical results for increasing values of $\mathrm{Pe}$ in terms of the dimensionless bulk temperature $\theta_{b}(\zeta)$ and wall temperature $\theta_{w}(\zeta)$ for the constant wall heat flux function.

The wall temperature $\theta_{w}(\zeta)$, accounting for the temperature jump induced by the rarefaction effect, is given by 


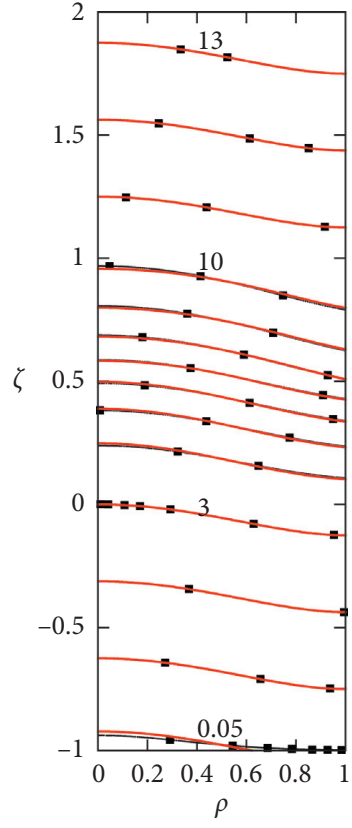

(a)

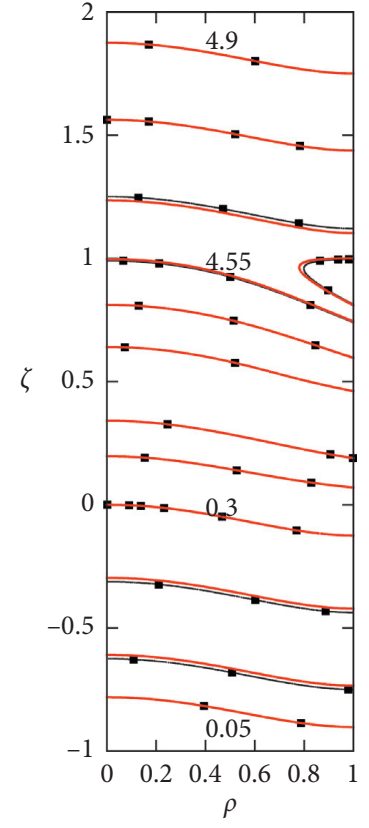

(b)

Figure 3: Contour plot of the dimensionless temperature $\theta(\rho, \zeta)$ for the triangular wall heat flux for $\mathrm{Pe}=100, \alpha=0.02$, and $\beta^{-}=\beta^{+}=1$. Comparison between numerical results (black lines with dots) and analytical results (continuous red lines). (a) $\mathrm{Br}=0.1, \mathrm{Kn}=0.0$; (b) $\mathrm{Br}=0.01, \mathrm{Kn}=0$. Labels indicate some reference temperature values.

$$
\begin{aligned}
\theta_{w}(\zeta) & =\theta(1, \zeta)+\left.2 b \operatorname{Kn} \frac{\partial \theta}{\partial \rho}\right|_{\rho=1}=\theta_{b}(\zeta)+\phi(1, \zeta)+2 b \operatorname{Kn} F_{w}(\zeta) \\
b & =\frac{2-F_{T}}{F_{T}} \frac{2 \gamma}{\gamma+1} \frac{1}{\operatorname{Pr}}
\end{aligned}
$$

where $F_{T}=1$ (thermal accommodation coefficient), $\gamma=1.4$, and $\operatorname{Pr}=0.7$ are assumed to be the typical values for air [26-28].

It can be observed that model predictions for $\theta_{b}(\zeta)$ are actually very accurate in the whole range of Peclet values $\mathrm{Pe} \in\left[10^{-2}, 10^{5}\right]$ because (1), at low Pe values, the simplifying assumption $\left(\partial^{2} \phi / \partial \zeta^{2}\right) \ll\left(\mathrm{d}^{2} \theta_{b} / \mathrm{d} \zeta^{2}\right)$ holds true and $(2)$, at high Pe values, the entire axial conduction term $\left(\partial^{2} \theta / \partial \zeta^{2}\right)$ becomes negligible with respect to the axial convective contribution $(\operatorname{Pe} \alpha / 4) v(\rho)(\partial \theta / \partial \zeta)[38-40]$.

Model predictions for the wall temperature $\theta_{w}$ are accurate for small-intermediate values of $\mathrm{Pe}, \mathrm{Pe} \leq 100$. Indeed, the analytical solution for $\theta_{w}$ follows quite closely the numerical solution also for $\mathrm{Pe}=10^{3}$, but small errors (related to the simplifying assumption equation (11)fd11 that fails at $\zeta=0$ and $\zeta=1$ for the uniform wall heat flux) are not negligible and are amplified when focusing on the spatial behaviour of local Nusselt number close to $\zeta=0,1$.

3.2. Local Nusselt Number. The local Nusselt number $N u(\zeta)$ in the heating section $(0 \leq \zeta \leq 1)$ attains the form

$$
\begin{aligned}
N u(0 \leq \zeta \leq 1) & =\frac{2 q_{w}}{\theta_{w}-\theta_{b}}=\frac{2 q_{w}(\zeta)}{\phi(1, \zeta)+2 b \mathrm{Kn} q_{w}(\zeta)} \\
& =\frac{2 q_{w}(\zeta)}{\left[q_{w}(\zeta)((1 / 4)+(\chi / 12)+2 b \mathrm{Kn})+\left(8 \mathrm{Br} \chi^{2}(1+\chi)+g(\zeta,(\mathrm{Pe} / \alpha)) \chi\right)((1 / 12)+(\chi / 24))\right]}
\end{aligned}
$$

and is a function solely of the ratio $\mathrm{Pe} / \alpha$ and not of $\mathrm{Pe}$ and $\alpha$ separately. Moreover, the local Nusselt number is independent of the lengths $\beta^{-}$and $\beta^{+}$of the upstream and downstream thermally insulated regions.

The two limiting cases $N u_{0}$ for $(\mathrm{Pe} / \alpha) \longrightarrow 0$ (no axial convection) and $N u_{\infty}$ for $(\mathrm{Pe} / \alpha) \longrightarrow \infty$ (Nusselt based on the fully developed temperature profile $\theta_{\infty}$ ) can be easily recovered from (34)fd 35 by considering that

$$
\begin{aligned}
\lim _{\mathrm{Pe} / \alpha \longrightarrow 0} g(\zeta, \mathrm{Pe} / \alpha) & =0, \\
\lim _{\mathrm{Pe} / \alpha \longrightarrow \infty} g(\zeta, \mathrm{Pe} / \alpha) & =q_{w}(\zeta),
\end{aligned}
$$




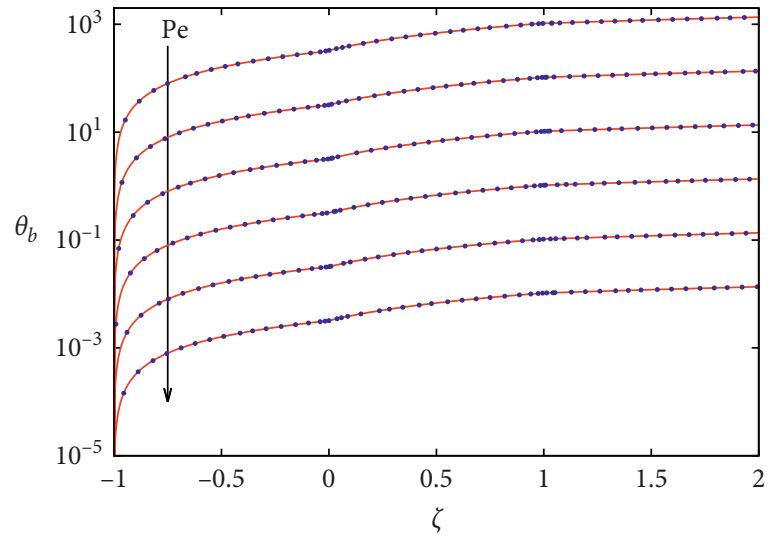

(a)

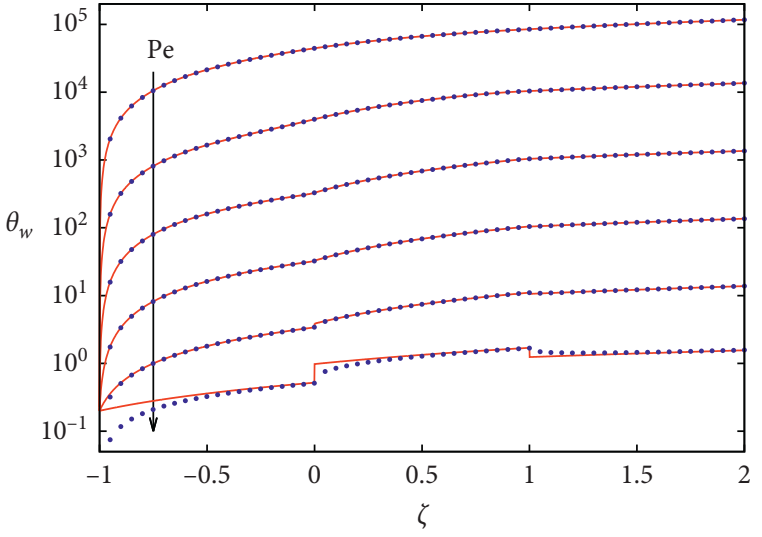

(b)

Figure 4: Comparison between numerical (blue dots) and analytical results (red continuous lines) for the constant wall heat flux $q_{w}(\zeta)=1$, $\mathrm{Br}=0.1, \mathrm{Kn}=0.0, \alpha=0.02, \beta^{-}=\beta^{+}=1$. (a) $\theta_{b}$ versus $\zeta$ for $\mathrm{Pe}=10^{0}, 10^{1}, 10^{2}, 10^{3}, 10^{4}, 10^{5}$; (b) $\theta_{w}$ versus $\zeta$ for $\mathrm{Pe}=10^{-2}$, $10^{-1}, 10^{0}, 10^{1}, 10^{2}, 10^{3}$. The arrows indicate increasing values of Pe.

thus obtaining

$$
\begin{aligned}
N u_{0} & =\frac{2 q_{w}(\zeta)}{\left[q_{w}(\zeta)((1 / 4)+(\chi / 12)+2 b \mathrm{Kn})+8 \mathrm{Br} \chi^{2}(1+\chi)((1 / 12)+(\chi / 24))\right]}, \\
N u_{\infty} & =\frac{2 q_{w}(\zeta)}{\left[q_{w}(\zeta)\left((1 / 4)+(\chi / 6)+\left(\chi^{2} / 24\right)+2 b \mathrm{Kn}\right)+8 \mathrm{Br} \chi^{2}(1+\chi)((1 / 12)+(\chi / 24))\right]} .
\end{aligned}
$$

Figures 5(a)and 5(b) show the comparison between numerical results (curves with dots) and analytical results for the local Nusselt number $N u(\zeta)$ for low-intermediate values of $P e=10^{-2}, 10^{-1}, 10^{0}, 10^{1}$ for the triangular wall heat flux (continuous at $\zeta=0,1$ ) and for the uniform wall heat flux (discontinuous at $\zeta=0,1$ ). The analytical solution is very accurate for the triangular function in the whole $\zeta$ range while deviations from the numerical solution can be observed for $\zeta<0.015$ and $\zeta>0.99$ for the uniform wall heat flux due to the simplifying assumption (11)fd11 that fails at discontinuity points $\zeta=0,1$.

However, the low accuracy of the analytical solution close to $\zeta=0,1$ for a discontinuous wall heat flux has a very small impact of the average Nusselt number:

$$
\langle N u\rangle=\int_{0}^{1} N u\left(\zeta^{\prime}\right) \mathrm{d} \zeta^{\prime}
$$

Figures 6(a)and 6(b) show the excellent agreement between numerical and analytical results for $\langle N u\rangle$ as a function of $\mathrm{Pe}$ for the exponential wall heat flux function (discontinuous at $\zeta=0,1$ ) in the presence of dissipation $\mathrm{Br}>0$, for constant $n=0$, axially increasing $(n<0)$ and decreasing $(n>0)$ wall heat flux. All data refers to an aspect ratio $\alpha=0.02$ (long-thin channel and long preheating section) and show an excellent agreement with numerical data up to $P e \simeq 20-40$.

\section{Limits of Validity of the Analytical Solution}

The analytical expression proposed is reliable also for larger values of the aspect ratio (finite-length channel). Figure 7 shows the comparison between numerical and analytical results for $\alpha=0.02$ and $\alpha=0.1$.

It can be observed that different curves, corresponding to different values of the aspect ratio, saturate towards the same limiting value, corresponding to the average Nusselt number $\left\langle N u_{\infty}\right\rangle$ evaluated on the basis of the fully developed temperature profile $\theta_{\infty}$. However, the larger is the value of $\alpha$, the smaller is the range of validity, in terms of Pe values, of the fully developed profile, and the influence of the thermal developing region must be necessarily accounted for (like in the analytical solution proposed) in order to have an accurate estimate of the average Nusselt number.

This observation becomes more evident by plotting the same data as in Figure 7 as a function of $\mathrm{Pe} / \alpha$ instead of $\mathrm{Pe}$ (see Figure 8(a)).

Indeed, numerical (and analytical) results for $\langle N u\rangle$ collapse onto an invariant curve when plotted as function of $\mathrm{Pe} / \alpha$ for low-intermediate Peclet values $\mathrm{Pe}<100$; see Figure $8(\mathrm{~A})$, and the asymptotic behaviour sets for $\mathrm{Pe} / \alpha \simeq 10^{2}$.

On the other hand, for high Peclet values, streamwise conduction becomes negligible and numerical results for $\langle N u\rangle$, for different aspect ratios, collapse onto a unique invariant curve for $(\mathrm{Pe} \alpha / 4)>10^{-1}$ when plotted as a function of $(\operatorname{Pe} \alpha / 4)$ (see Figure 8(b)). 


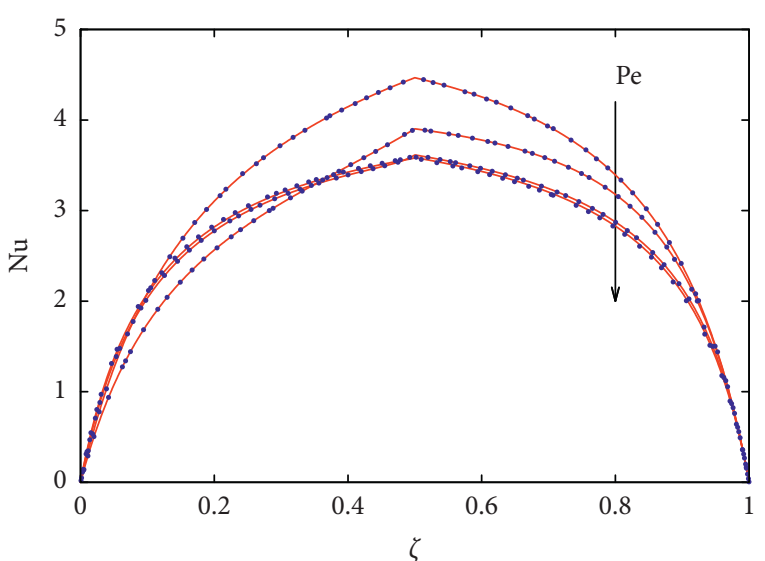

(a)

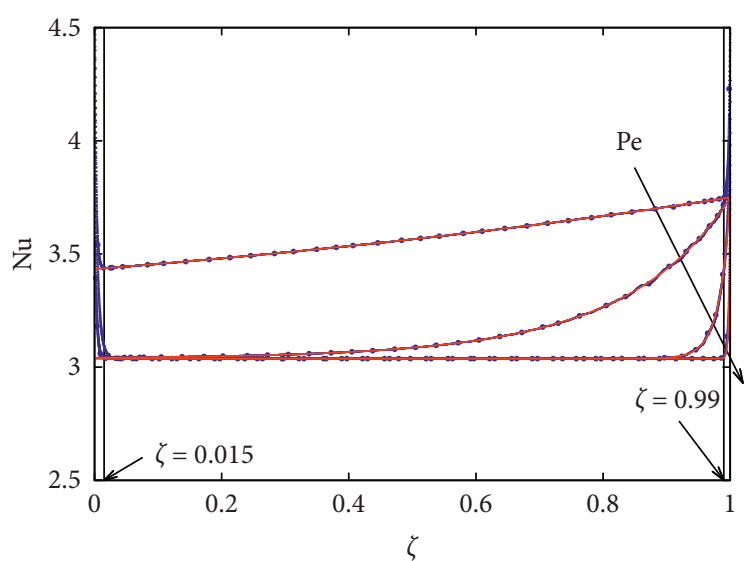

(b)

Figure 5: Comparison between numerical (blue curves with dots) and analytical results (red continuous lines) for $N u(\zeta)$ versus $\zeta$. The arrows indicate increasing values of $\mathrm{Pe} . \mathrm{Br}=0.1, \mathrm{Kn}=0.0, \alpha=0.02, \beta^{+}=\beta^{-}=1, P e=10^{-2}, 10^{-1}, 10^{0}, 10^{1}$. (a) Triangular wall heat flux. (b) Uniform wall heat flux.



(a)

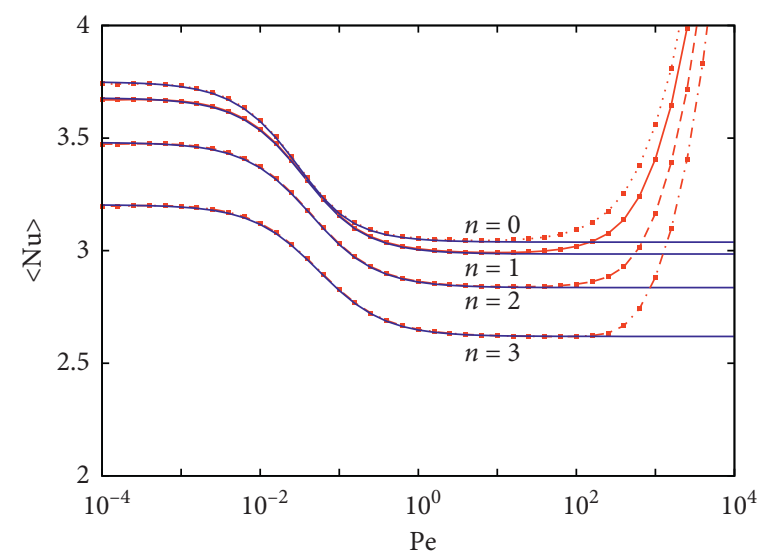

(b)

Figure 6: Comparison between numerical (red curves with dots) and analytical results (blue continuous lines) for the average Nusselt $\langle N u\rangle$ as a function of $P e$ for the exponential wall heat flux function $q_{w}(\zeta)=\left(n e^{-n \zeta} / 1-e^{-n}\right), \mathrm{Br}=0.1, \mathrm{Kn}=0.0, \alpha=0.02, \beta^{+}=\beta^{-}=2$. (a) Constant wall heat flux $n=0$ and exponentially increasing wall heat flux $n=-1,-2,-3,-5$. (b) Constant wall heat flux $n=0$ and exponentially decreasing wall heat flux $n=1,2,3$.



Figure 7: Comparison between numerical (red dotted lines) and analytical results (blue continuous lines) for the average Nusselt $\langle N u\rangle$ as a function of $\mathrm{Pe}$ for $q_{w}(\zeta)=1$ with $\mathrm{Br}=0$ and $\mathrm{Br}=0.1, \mathrm{Kn}=0$. Two different values of $\alpha$ are considered, $\alpha=0.02$ and $\alpha=0.1$. The arrows indicate increasing values of $\alpha$. 


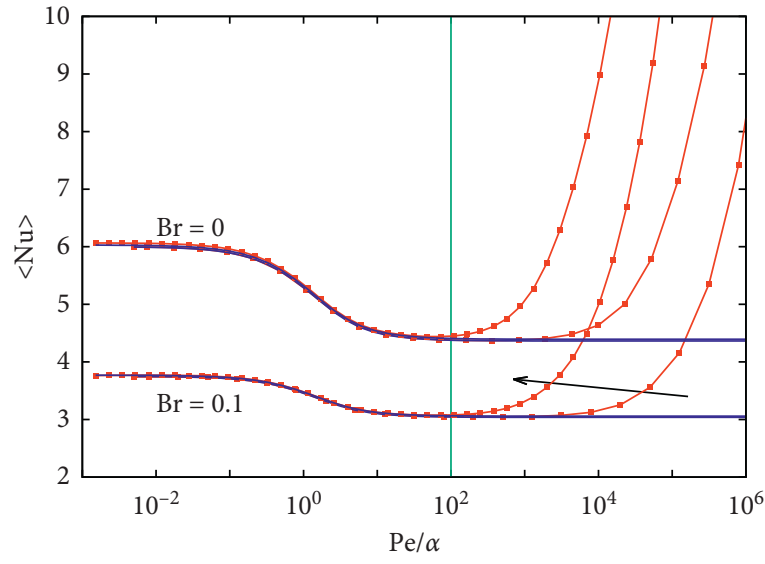

(a)

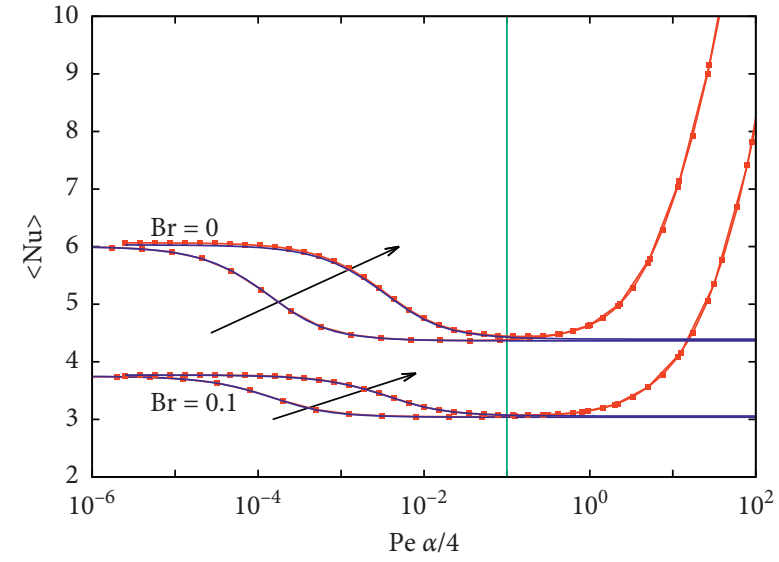

(b)

Figure 8: Comparison between numerical (red dotted lines) and analytical results (blue continuous lines) for the average Nusselt $\langle N u\rangle$ as a function of $\mathrm{Pe} / \alpha$ (a) and $\mathrm{Pe} \alpha / 4$ (b) for $q_{w}(\zeta)=1$. Two different values of $\alpha$ are considered, $\alpha=0.02$ and $\alpha=0.1$. Figures 8 (a)-8(b) refer to $\mathrm{Br}=0$ and $\mathrm{Br}=0.1, \mathrm{Kn}=0$. Reference values (vertical lines) are $P e / \alpha=10^{2}$ and $P e \alpha / 4=10^{-1}$. The arrows indicate increasing values of $\alpha$.

From these observations, it follows that the analytical solution proposed is actually reliable for $0<(\mathrm{Pe} \alpha / 4) \leq 10^{-1}$ that implies for $0<P e \leq\left(4 \cdot 10^{-1} / \alpha\right)$ (see Figure $\left.8(\mathrm{~b})\right)$. On the other hand, the solution $\left\langle N u_{\infty}\right\rangle$ based on the fully developed temperature profile $\theta_{\infty}$ is valid only in the range $(P e / \alpha) \geq 10^{2}$ and $(P e \alpha / 4) \leq 10^{-1}$ that implies $10^{2} \alpha \leq P e \leq\left(4 \cdot 10^{-1} / \alpha\right)$.

Figure 9 shows the range of validity, in terms of $P e$ and $\alpha$ values, of the analytical solution proposed in (34)fd35 (region with vertical bars) and of that based on the fully developed temperature profile $N u_{\infty}$ (triangular region indicated by arrows), the latter reducing to an empty set for $\alpha \longrightarrow 0.063$.

Data reported in Figures 8(a), 8(b), and 9 refer to a case in which the rarefaction effect is not present since $K n=0$. The same analysis can be performed by including the effect of rarefaction $(K n \neq 0)$. The region of stability of the analytical solution for $K n \neq 0$ almost coincides with that for $K n=0$.

The next section analyzes the influence of different parameters and of different wall heat flux functions on the average Nusselt number $\left\langle N u_{\infty}\right\rangle$, focusing exclusively on the range of validity of the analytical expression equation (34)fd35.

\section{Influence of Wall Heat Flux Function and Transport Parameters $\mathrm{Br}$ and $\mathrm{Kn}$}

From a preliminary analysis of data reported in Figures 6(a)6(b), it can be observed that, in presence of dissipation, that is, $B r>0$, the uniform wall heat flux function represents the best heating protocol (larger value of $\left\langle N u_{\infty}\right\rangle$ for low-intermediate values of $\mathrm{Pe}$ ) with respect to both monotonically increasing or monotonically decreasing (exponential) wall heat flux functions.

For the axially increasing wall heat flux, the average Nusselt number exhibits a minimum for $P e \simeq 5 \alpha$ (see Figure 6(a)) and the minimum is more pronounced for increasing values of $n$, corresponding to an increasing

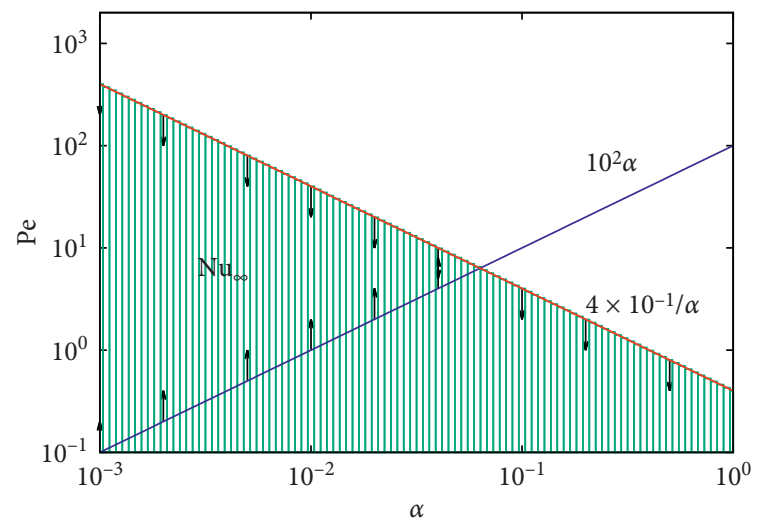

FIGURE 9: Region of validity of the analytical solution equation (35) (region with vertical bars below the red curve $P e=\left(4 \cdot 10^{-1} / \alpha\right)$ ) and of $N u_{\infty}$ (triangular region below the red curve $P e=\left(4 \cdot 10^{-1} / \alpha\right)$ and above the blue curve $\left.\mathrm{Pe}=10^{2} \alpha\right)$.

amount of energy furnished close to the channel outlet $\zeta=1$. For $\mathrm{Br}>0$, no minimum is observed for decreasing wall heat flux functions (see Figure 6(b)).

For a constant wall heat function $q_{w}=1,\left\langle N u_{\infty}\right\rangle$ is a monotonically decreasing function of $P e / \alpha$, attaining values in the range $\left[\left\langle N u_{\infty}\right\rangle,\left\langle N u_{0}\right\rangle\right]$.

Figures $10(\mathrm{a})$ and $10(\mathrm{~b})$ show the upper bound $\left\langle N u_{0}\right\rangle$ and the lower bound $\left\langle N u_{\infty}\right\rangle$ as a function of $K n$ for $B r=0,0.05,0.1$. The effect of dissipation is to lower $\langle N u\rangle$ by decreasing both the upper and lower bound. The effect of $K n$ is to monotonically lower both the upper and the lower bounds in the absence of dissipation $\mathrm{Br}=0$ while, for $\mathrm{Br}>0$, the upper and lower bounds exhibit a maximum as a function of $K n$.

In the absence of dissipation, that is, $B r=0$, both $N u_{0}(\zeta)$ and $N u_{\infty}(\zeta)$ become constant and independent of the wall heat flux function (see (37) and (38) for $\mathrm{Br}=0$ ). For this reason, the values of $\left\langle N u_{0}\right\rangle$ and $\left\langle N u_{\infty}\right\rangle$ reported in Figures $10 \mathrm{~A}-\mathrm{B}$ for $\mathrm{Br}=0$ represent the asymptotic limits 


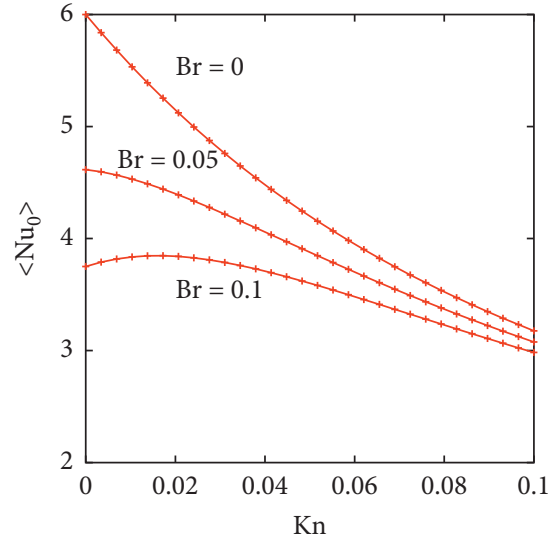

(a)

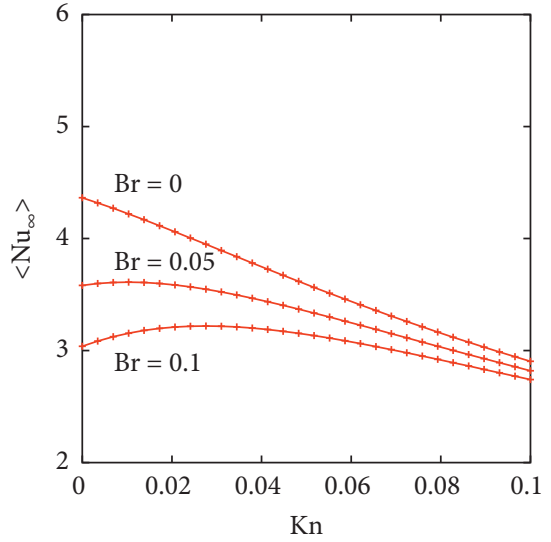

(b)

Figure 10: Upper bound $\left\langle N u_{0}\right\rangle$ and lower bound $\left\langle N u_{\infty}\right\rangle$ for $\langle N u\rangle$ as a function of $K n$ for $B r=0,0.05,0.1$. Constant wall heat flux $q_{w}(\zeta)=1$.

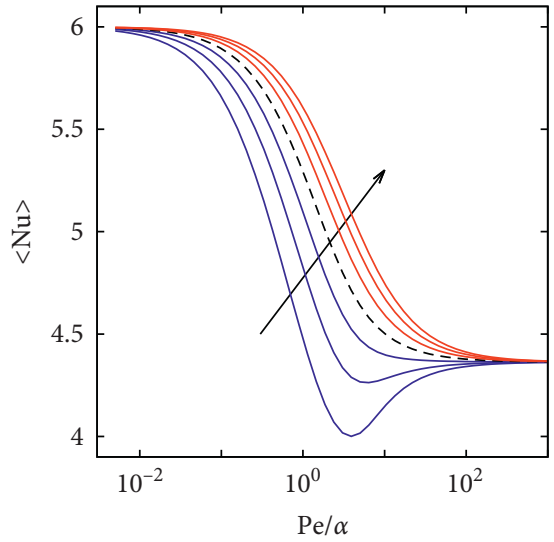

(a)

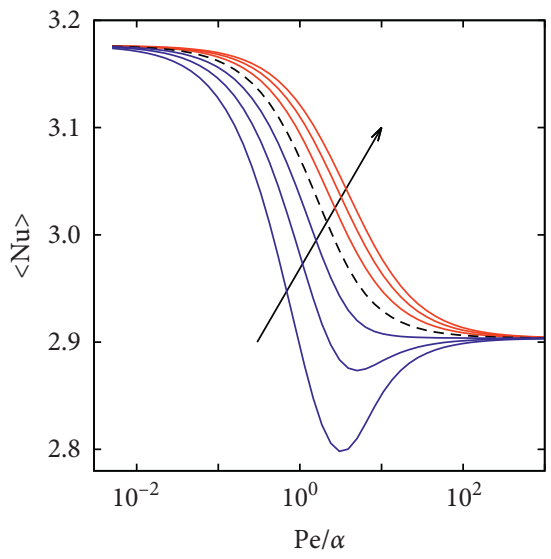

(b)

Figure 11: $\langle N u\rangle$ versus $P e / \alpha$ for $q_{w}(\zeta)=\left(n e^{-n \zeta}\right) /\left(1-e^{-n}\right)$. No viscous dissipation $B r=0$. The arrow indicates increasing values of $n$. $n=0$ (black broken line) corresponds to a constant function $q_{w}(\zeta)=1$. The blue curves correspond to increasing wall heat flux. The red curves correspond to decreasing wall heat flux. (a) $K n=0$; (b) $K n=0.1$.

valid for any other wall heat flux function. However, $\left\langle N u_{\infty}\right\rangle$ may not be the minimum value attained by $\langle N u\rangle$ because it may exhibit a minimum for intermediate value of $P e$, depending on the wall heat flux function.

In order to investigate the role of the wall heat flux function, the following analysis focuses on the exponential function equation (31)fd31 that represents a decreasing function of $\zeta$ for $n>0$ and an increasing function of $\zeta$ for $n<0$. For $n=0$, it reduces to the constant function $q_{w}=1$. For this exponential heat function, $\langle N u\rangle$ can be evaluated analytically for $\mathrm{Br}=0$, thus obtaining

$$
\begin{aligned}
\langle N u\rangle & =\frac{2}{(n+(P e / \alpha)) A(K n)+(P e / \alpha) B(K n)}\left[n+\frac{P e}{\alpha}+\log \left(1+\frac{\left(1-e^{-(n-(P e / \alpha))}\right)(P e / \alpha) B(K n)}{(n+(P e / \alpha)) A(K n)}\right)\right], \\
A(K n) & =\left(\frac{1}{4}+\frac{\chi}{12}+2 b K n\right), \\
B(K n) & =\left(\frac{\chi}{12}+\frac{\chi^{2}}{24}\right) .
\end{aligned}
$$




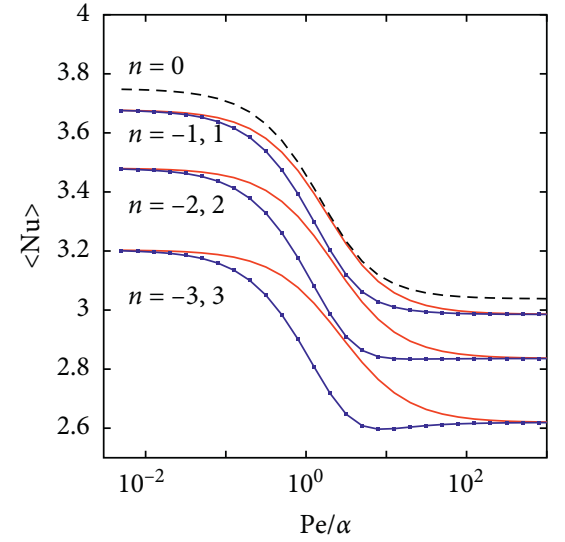

(a)

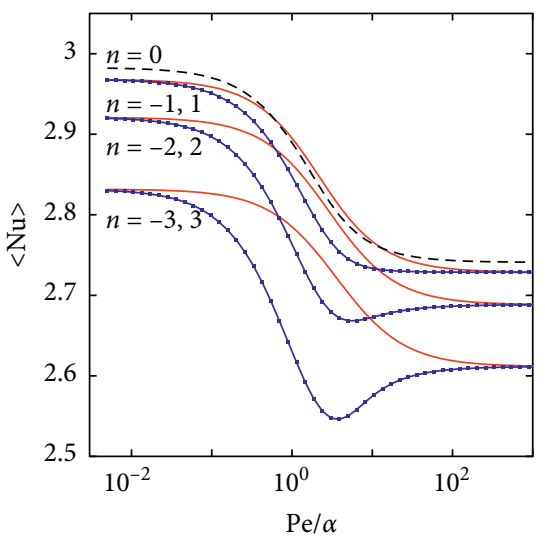

(b)

Figure 12: $\langle N u\rangle$ versus $P e / \alpha$ for $q_{w}(\zeta)=\left(n e^{-n \zeta}\right) /\left(1-e^{-n}\right)$ with viscous dissipation $B r=0.1 . n=0$ (black broken line) corresponds to a constant function $q_{w}(\zeta)=1$. Blue lines with dots correspond to axially increasing wall heat flux, $n<0$. Red continuous lines correspond to axially decreasing wall heat flux, $n>0$. (a) $K n=0$; (b) $K n=0.1$.

Figures 11(a)and 11(b) show the behaviour of $\langle N u\rangle$ as a function of $P e / \alpha$ for $B r=0$ and for $K n=0,0.1$. The arrow indicates increasing values of $n$, in the range $n \in[-3,3]$. The larger is $n$, the larger is the value of $\langle N u\rangle$ for low-intermediate values of $P e$. For $n<0,\langle N u\rangle$ exhibits a neat minimum.

Therefore, in the absence of dissipation, a decreasing wall heat flux function $(n>0)$ has to be preferred to a constant heat flux function and the larger part of energy must be provided at the entrance of the heating section. On the contrary, in the presence of viscous dissipation, a constant wall heat flux represents the best heating protocol, as shown in Figures 6(a)-6(b) and Figures 12(a) and 12(b) for Br $=0.1$. High values of $K n$ amplify differences between increasing and decreasing wall heat flux functions.

\section{Conclusions}

The paper proposes an analytical solution for the extended Graetz-Nusselt problem in finite-length microchannels, including the effects of rarefaction, streamwise conduction, and viscous dissipation. The solution takes into account the presence of a thermally insulated upstream section. Different wall heat functions are analyzed.

A classical approach to the problem (see Jeong and Jeong $[28,29]$ and Tunc and Bayazitoglu [30]) suggested solving the nonhomogeneous energy balance equation for the dimensionless temperature $\theta(\rho, \zeta)$ by setting $\theta=\theta_{1}(\rho, \zeta)+\theta_{\infty}(\rho)$, where $\theta_{\infty}$ is the fully developed temperature profile and $\theta_{1}$ satisfies an homogeneous partial differential equation that can be solved by the method of separation of variables or by integral transform.

In the present case, the analysis focuses on finite-length channels. Therefore, a different "decoupling" of the problem is proposed leading to a more convenient way to express the temperature profile $\theta$ as the linear combination of the bulk temperature $\theta_{b}(\zeta)$ and of the auxiliary function $\phi(\rho, \zeta)$, accounting for the dependence of the temperature on the radial coordinate $\rho$ and slowly varying with the axial coordinate $\zeta$. This represents the peculiarity and the strength of our approach to the problem, valid for low-intermediate Peclet values. A similar approach can be adopted to deal with the same transport problem with prescribed wall temperature condition (instead of a prescribed wall heat flux). A Dirichlet boundary condition would require a different nondimensionalization of the transport equation and the solution of an inverse problem; that is, given the analytic expression of the wall temperature for prescribed wall heat flux, find the wall heat flux function $q_{w}(\zeta)$ that guarantees the required wall temperature profile. This problem is already under investigation.

The range of validity of the analytical solution is investigated by comparing analytical and numerical results obtained with finite elements method. By analyzing different wall heating functions, different values of $P e$, and different channel aspect ratios $\alpha$, it is possible to conclude that the analytical solution is reliable for $\mathrm{Pe}<(1 / \alpha)$.

The influence of various transport parameters, that is, $\mathrm{Br}$ and $K n$, is analyzed in detail.

The solution proposed is valid in the whole range of Brinkman values considered in the microfluidic literature, that is, $B r \leq 0.1$, and therefore valid also in the absence of dissipation effects. Moreover the solution is valid for slip $(K n>0)$ as well as for nonslip flows $(K n=0)$ and therefore also for viscous fluids for which dissipation effects may not be negligible.

Actually, a value of Brinkman less than $0.1-0.2$ is rather realistic for microfluidic applications and compatible with Peclet numbers less than 100.

Performances of different wall heat flux functions have been analyzed in terms of the averaged Nusselt number $\langle N u\rangle$. In the absence of viscous dissipation, the best heating protocol is a decreasing wall heat flux function, where the larger part of energy is furnished at the entrance of the downstream-heated section.

In the presence of dissipation, that is, $B r>0$, the best heating protocol is a uniform wall heat flux and decreasing wall heat flux functions have to be preferred to increasing wall heat flux functions. 
In Appendix A the analytical solution proposed for circular microchannels is extended to the case of flat $2-\mathrm{d}$ rectangular microchannels. The influence of the cross section aspect ratio for rectangular microchannels (three-dimensional problem) on slip-flow heat transfer [41, 42] is not investigated.

\section{Appendix}

\section{A. Flat Microchannels}

The analytical solution proposed for circular microchannels (microtubes) can be extended to flat rectangular microchannels. Let $2 \mathrm{H}$ be channel height (the distance between the two flat plates) and $W$ the channel width with $W \gg H$, such that the hydraulic diameter $D_{h} \longrightarrow 4 H$.

The laminar velocity profile, depending exclusively on the cross section coordinate $\eta=(y / H) \in[-1,1]$ and satisfying the slip boundary condition $V_{z}(y)=\mp[(2-F) /$ $F] \lambda\left(\partial V_{z} / \partial y\right)$ at $y= \pm H$ attains the form (for $F=1$ )

$$
\begin{aligned}
v(\eta) & =\frac{V_{z}(\eta)}{U}=\frac{3}{2} \frac{\left(1-\eta^{2}+4 K n\right)}{1+6 K n}=\frac{3}{2}\left(1-\eta^{2}\right) \chi+(1-\chi), \\
K n & =\frac{\lambda}{2 H} \\
\chi & =\frac{1}{1+6 K n} .
\end{aligned}
$$

The transport equation and boundary conditions for the dimensionless temperature $\theta=\left(T-T_{0}\right) k /\left(Q_{a v} H\right)$ read as

$$
\begin{aligned}
-\frac{P e \alpha}{4} v(\eta) \frac{\partial \theta}{\partial \zeta}+\frac{\partial^{2} \theta}{\partial \eta^{2}}+\frac{\alpha^{2}}{4} \frac{\partial^{2} \theta}{\partial \zeta^{2}}+18 B r \chi^{2} \eta^{2} & =0 \\
\left.J_{c}\right|_{\zeta=-\beta^{-}} & =0, \\
\left.\left(\frac{\partial}{\partial \zeta} \int_{-1}^{1} J_{c}(\eta, \zeta) \mathrm{d} \eta\right)\right|_{\zeta=1+\beta^{+}} & =12 B r \chi^{2},
\end{aligned}
$$

$$
\begin{aligned}
& \left.\frac{\partial \theta}{\partial \eta}\right|_{\eta=0}=0, \\
& \left.\frac{\partial \theta}{\partial \eta}\right|_{\eta=1}=F_{w}(\zeta)= \begin{cases}0, & \text { for }-\beta^{-} \leq \zeta<0, \\
q_{w}(\zeta), & \text { for } 0 \leq \zeta \leq 1, \\
0, & \text { for } 1<\zeta \leq 1+\beta^{+},\end{cases}
\end{aligned}
$$

where

$$
J_{c}(\eta, \zeta)=\frac{P e \alpha}{4} v(\eta) \theta(\eta, \zeta)
$$

and all the dimensionless parameters are the same as defined for microtubes by simply replacing the diameter $D$ with the channel height $2 \mathrm{H}$

$$
\begin{aligned}
P e & =\frac{U 2 H}{\left(k / \rho_{f} c_{p}\right)}, \\
B r & =\frac{\mu U^{2}}{Q_{a v} 2 H}, \\
\alpha & =\frac{2 H}{L}, \\
\beta^{-} & =\frac{L^{-}}{L^{\prime}}, \\
\beta^{+} & =\frac{L^{+}}{L} .
\end{aligned}
$$

The analytical solution $\theta(\eta, \zeta)$ can be sought as the linear combination of the bulk temperature $\theta_{b}(\zeta)$ and of an auxiliary function $\phi(\eta, \zeta)$ satisfying the conditions

$$
\theta_{b}(\zeta)=\frac{1}{2} \int_{-1}^{1} \theta v(\eta) \mathrm{d} \eta
$$

$$
\frac{1}{2} \int_{-1}^{1} \phi(\eta, \zeta) v(\eta) \mathrm{d} \eta=0 .
$$

By assuming

$$
\begin{gathered}
\frac{\partial \phi}{\partial \zeta} \ll \frac{d \theta_{b}}{d \zeta}, \\
\frac{\partial^{2} \phi}{\partial \zeta^{2}} \ll \frac{d^{2} \theta_{b}}{d \zeta^{2}},
\end{gathered}
$$

the transport equation (A.2) and the boundary conditions equations (A.3) and (A.4) can be rewritten in terms of $\theta_{b}$ and $\phi$ as follows:

$$
\begin{aligned}
-\frac{P e \alpha}{4} v(\eta) \frac{d \theta_{b}}{d \zeta}+\frac{\partial^{2} \phi}{\partial \eta^{2}}+\frac{\alpha^{2}}{4} \frac{d^{2} \theta_{b}}{d \zeta^{2}}+18 B r \chi^{2} \eta^{2}=0 \\
\left.\theta_{b}\right|_{\zeta=-\beta^{-}}=0, \\
\frac{P e \alpha}{4} \frac{\mathrm{d} \theta_{b}}{\left.\mathrm{~d} \zeta\right|_{\zeta=1+\beta^{+}}}=6 B r \chi^{2}, \\
\left.\frac{\partial \phi}{\partial \eta}\right|_{\eta=0}=0, \\
\left.\frac{\partial \phi}{\partial \eta}\right|_{\eta=1}=F_{w}(\zeta) .
\end{aligned}
$$

By integrating (A.2) over the entire cross section and by enforcing the boundary conditions equation (A.4) at $\eta=0,1$, one obtains the following equation for the bulk temperature $\theta_{b}(\zeta)$ : 


$$
\begin{aligned}
-\frac{P e \alpha}{4} \frac{\mathrm{d} \theta_{b}}{\mathrm{~d} \zeta}+\frac{\alpha^{2}}{4} \frac{\mathrm{d}^{2} \theta_{b}}{\mathrm{~d} \zeta^{2}}+6 B r \chi^{2}+F_{w}(\zeta) & =0, \\
\left.\theta_{b}\right|_{\zeta=-\beta^{-}} & =0, \\
\left.\frac{P e \alpha}{4} \frac{\mathrm{d} \theta_{b}}{\mathrm{~d} \zeta}\right|_{\zeta=1+\beta^{+}} & =6 B r \chi^{2},
\end{aligned}
$$

which can be solved analytically, thus obtaining

$$
\begin{aligned}
\theta_{b}(\zeta)= & \frac{4}{P e^{2}}[g(\zeta,(P e / \alpha))-g(-\beta) \\
& +\frac{P e}{\alpha}\left(I F_{w}(\zeta)+6 B r \chi^{2}\left(\left(z+\beta^{-}\right)\right)\right] \\
g(\zeta<0,(P e / \alpha))= & \frac{P e}{\alpha} e^{(P e \zeta) / \alpha} \int_{0}^{1} e^{\left(-P e \zeta^{\prime}\right) / \alpha} q_{w}\left(\zeta^{\prime}\right) \mathrm{d} \zeta^{\prime} \\
g(0 \leq \zeta \leq 1,(P e / \alpha))= & \frac{P e}{\alpha} e^{(P e \zeta) / \alpha} \int_{\zeta}^{1} e^{\left(-P e \zeta^{\prime}\right) / \alpha} q_{w}\left(\zeta^{\prime}\right) \mathrm{d} \zeta^{\prime} \\
g(\zeta>1,(P e / \alpha))= & 0 \\
I F_{w}(\zeta<0)= & 0 \\
I F_{w}(0 \leq \zeta \leq 1)= & \int_{\zeta}^{1} q_{w}\left(\zeta^{\prime}\right) \mathrm{d} \zeta \\
I F_{w}(\zeta>1)= & 1 .
\end{aligned}
$$

By substituting the expression for the bulk temperature into the balance equation (A.9), one obtains the following $\operatorname{PDE}$ for $\phi(\eta, \zeta)$ :

$$
\begin{aligned}
\frac{\partial^{2} \phi}{\partial \eta^{2}}= & \left(g(\zeta, P e / \alpha)+6 B r \chi^{2}\right)((v(\rho)-1) \\
& +\left(F_{w}(\zeta)+6 B r \chi^{2}\right)-18 B r \chi^{2} \eta^{2}
\end{aligned}
$$

which can be solved analytically for $\phi(\eta, \zeta)$, thus obtaining

$$
\begin{aligned}
\phi(\eta, \zeta)= & \frac{\eta^{2}}{4}\left[6 B r \chi^{2}(2+\chi)+g(\zeta,(P e / \alpha)) \chi+2 F_{w}(\zeta)\right]+ \\
& -\frac{\eta^{4}}{8}\left[6 B r \chi^{2}(2+\chi)+g(\zeta,(P e / \alpha)) \chi\right]+C(\zeta) \\
C(\zeta)= & -\frac{7}{20} B r \chi^{2}(2+\chi)-\frac{7}{120} g(\zeta,(P e / \alpha)) \chi-\frac{F_{w}(\zeta)}{6} .
\end{aligned}
$$

The analysis of the exact limits of validity of the analytical solution for a flat microchannel will be developed elsewhere, by following the same approach presented for microtubes.

\section{B. List of Symbols}

$B r=\mu U^{2} /\left(Q_{a v} D\right):$ Brinkman number,

$c_{p}$ : fluid specific heat,

$D$ : channel diameter,

$F, F_{T}$ : tangential momentum and thermal accommodation coefficients,

$g(\zeta,(P e / \alpha))$ : integral function, (19)-(21),

$h$ : heat transfer coefficient,

$I F_{w}(z)$ : integral wall heat flux function, (22)-(23),

$k$ : thermal conductivity,

$K n=(\lambda / D):$ Knudsen number,

$L$ : length of the channel heated section,

$L^{-}, L^{+}$: upstream and downstream insulated channel lengths,

$N u=(h D / k):$ Nusselt number,

$\langle N u\rangle$ : average Nusselt number,

$N u_{0}$ : Nusselt number for $(\mathrm{Pe} / \alpha) \longrightarrow 0$,

$N u_{\infty}$ : Nusselt number for $\alpha \longrightarrow \infty$,

$P e=U D /\left(k / \rho_{f} c_{p}\right)$ : cross-sectional Peclet number,

$q_{w}(\zeta)=\left(Q_{w}(z / L)\right) / Q_{\mathrm{av}}$ : dimensionless wall heat flux function,

$Q_{\mathrm{av}}$ : average wall heat flux,

$r, z$ : radial and axial coordinates,

$R$ : channel radius,

$T$ : temperature,

$T_{0}$ : inlet temperature at $z=-L^{-}$,

$U$ : average axial velocity,

$V_{z}(r / R), v(\rho)$ : dimensional and dimensionless axial velocity profile, equation (1).

\section{Greek symbols}

$\alpha=D / L:$ channel aspect ratio,

$\beta^{-}=L^{-} / L, \beta^{+}=L^{+} / L$ : dimensionless insulated upstream and downstream section lengths,

$\theta=\left(\left(T-T_{0}\right) k\right) /\left(Q_{\mathrm{av}} R\right)$ : dimensionless temperature,

$\theta_{b}(\zeta)$ : dimensionless bulk temperature,

$\lambda$ : molecular mean free path,

$\xi=z /(P e R)$ : dimensionless axial coordinate entering equation (29),

$\phi(\rho, \zeta)$ : auxiliary function, equation (9),

$\chi=1 /(1+8 K n)$

$\rho, \zeta$ : dimensionless radial and axial coordinates,

$\rho_{f}$ : fluid density.

\section{Data Availability}

The data used to support the findings of this study are available from the corresponding author upon request. 


\section{Conflicts of Interest}

The authors declare that there are no conflicts of interest regarding the publication of this paper.

\section{References}

[1] W. L. McCabe, J. C. Smith, and P. Harriott, Unit Operations of Chemical Engineering, Vol. 5, McGraw-Hill, New York, NY, USA, 1993.

[2] E. L. Cussler and E. L. Cussler, Diffusion: Mass Transfer in Fluid Systems, Cambridge University Press, Cambridge, UK, 2009.

[3] H. A. Stone, A. D. Stroock, and A. Ajdari, "Engineering flows in small devices," Annual Review of Fluid Mechanics, vol. 36, no. 1, pp. 381-411, 2004.

[4] T. M. Squires and S. R. Quake, "Microfluidics: fluid physics at the nanoliter scale," Reviews of Modern Physics, vol. 77, no. 3, pp. 977-1026, 2005.

[5] G. Groppi, A. Belloli, E. Tronconi, and P. Forzatti, "A comparison of lumped and distributed models of monolith catalytic combustors," Chemical Engineering Science, vol. 50, no. 17, pp. 2705-2715, 1995.

[6] H. Bruus, Theoretical Microfluidics, Vol. vol. 18, Oxford university press, Oxford, UK, 2008.

[7] R. Cotta, S. Kakaç, M. Mikhailov, F. V. Castellões, and C. Cardoso, "“Transient flow and thermal analysis in microfluidics," microscale heat transfer Fundamentals and applications," NATO Science Series II: Mathematics, Physics and Chemistry, vol. 193, pp. 175-196, 2005.

[8] A. Adrover and M. Nobili, "Release kinetics from oral thin films: theory and experiments," Chemical Engineering Research and Design, vol. 98, pp. 188-201, 2015.

[9] A. Adrover and A. Pedacchia, "Mass transfer through laminar boundary layer in microchannels with nonuniform cross section: the effect of wall shape and curvature," International Journal of Heat and Mass Transfer, vol. 60, pp. 624-631, 2013.

[10] R. B. Bird, W. E. Stewart, E. N. Lightfoot, and R. E. Meredith, "Transport phenomena," Journal of The Electrochemical Society, vol. 108, no. 3, pp. 78C-79C, 1961.

[11] R. M. Cotta and M. N. Özişik, "Transient forced convection in laminar channel flow with stepwise variations of wall temperature," The Canadian Journal of Chemical Engineering, vol. 64, no. 5, pp. 734-742, 1986.

[12] J. P. Lopes, S. S. S. Cardoso, and A. E. Rodrigues, "Bridging the gap between Graetz's and lévêque's analyses for mass/heat transfer in a channel with uniform concentration or flux at the wall," AIChE Journal, vol. 58, no. 6, pp. 1880-1892, 2012.

[13] A. Adrover and A. Pedacchia, "Mass/heat transfer through laminar boundary layer in axisymmetric microchannels with nonuniform cross section and fixed wall concentration/ temperature," International Journal of Heat and Mass Transfer, vol. 68, pp. 21-28, 2014.

[14] A. Adrover, "Laminar convective heat transfer across fractal boundaries," EPL (Europhysics Letters), vol. 90, no. 1, p. 14002, 2010.

[15] C. Tien, "Laminar heat transfer of power-law non-Newtonian fluid-the extension of Graet-Nusselt problem," The Canadian Journal of Chemical Engineering, vol. 40, no. 3, pp. 130-134, 1962.

[16] R. J. Nunge and W. N. Gill, "An analytical study of laminar counterflow double-pipe heat exchangers," AIChE Journal, vol. 12, no. 2, pp. 279-289, 1966.

[17] P. P. Valkó, “"Solution of the graetzâ€"brinkman problem with the laplace transform galerkin method," International
Journal of Heat and Mass Transfer, vol. 48, no. 9, pp. 18741882, 2005.

[18] M. L. Michelsen and J. Villadsen, "The graetz problem with axial heat conduction," International Journal of Heat and Mass Transfer, vol. 17, no. 11, pp. 1391-1402, 1974.

[19] A. Barletta and E. Zanchini, "On the laminar forced convection with axial conduction in a circular tube with exponential wall heat flux," Heat and Mass Transfer, vol. 30, no. 5, pp. 283-290, 1995.

[20] R. M. L. Coelho and A. Silva Telles, "Extended graetz problem accompanied by dufour and soret effects," International Journal of Heat and Mass Transfer, vol. 45, no. 15, pp. 31013110, 2002.

[21] H.-T. Lin and L.-K. Lin, "Similarity solutions for laminar forced convection heat transfer from wedges to fluids of any Prandtl number," International Journal of Heat and Mass Transfer, vol. 30, no. 6, pp. 1111-1118, 1987.

[22] M. Giona, A. Adrover, and S. Cerbelli, "Spectral analysis of the weighted laplacian in slip and no-slip flows," Phys. Rev. E, vol. 80, Article ID 066302, 2009.

[23] A. Adrover, S. Cerbelli, F. Garofalo, and M. Giona, "Convection-dominated dispersion regime in wide-bore chromatography: a transport-based approach to assess the occurrence of slip flows in microchannels," Analytical Chemistry, vol. 81, no. 19, pp. 8009-8014, Article ID 19739656, 2009.

[24] A. Adrover and S. Cerbelli, "Laminar dispersion at low and high peclet numbers in finite-length patterned microtubes," Physics of Fluids, vol. 29, no. 6, Article ID 062005, 2017.

[25] A. Adrover, S. Cerbelli, and M. Giona, "Taming axial dispersion in hydrodynamic chromatography columns through wall patterning," Physics of Fluids, vol. 30, no. 4, 042002 pages, 2018.

[26] B. Çetin, A. Gűvenç Yaziciog̃lu, and S. Kakaç, "Slip-flow heat transfer in microtubes with axial conduction and viscous dissipation - an extended Graetz problem," International Journal of Thermal Sciences, vol. 48, no. 9, pp. 1673-1678, 2009.

[27] C. Barbaros and B. Ozgur, "Evaluation of nusselt number for a flow in a microtube using second-order slip model," Thermal Science, vol. 15, pp. S103-S109, 2011.

[28] H.-E. Jeong and J.-T. Jeong, "Extended graetz problem including streamwise conduction and viscous dissipation in microchannel," International Journal of Heat and Mass Transfer, vol. 49, no. 13-14, pp. 2151-2157, 2006.

[29] H.-E. Jeong and J.-T. Jeong, "Extended graetz problem including axial conduction and viscous dissipation in microtube," Journal of Mechanical Science and Technology, vol. 20, no. 1, pp. 158-166, 2006.

[30] G. Tunc and Y. Bayazitoglu, "Heat transfer in microtubes with viscous dissipation," International Journal of Heat and Mass Transfer, vol. 44, no. 13, pp. 2395-2403, 2001.

[31] D. Hennecke, "Heat transfer by hagen-Poiseuille flow in the thermal development region with axial conduction," WÃ arme - und StoffÃ $1 / 4$ bertragung, vol. 1, no. 3, pp. 177-184, 1969.

[32] A. Barletta and E. Magyari, "Forced convection with viscous dissipation in the thermal entrance region of a circular duct with prescribed wall heat flux," International Journal of Heat and Mass Transfer, vol. 50, no. 1-2, pp. 26-35, 2007.

[33] D. C. Knupp, R. M. Cotta, and C. P. Naveira-Cotta, "Heat transfer in microchannels with upstream-downstream regions coupling and wall conjugation effects," Numerical Heat Transfer, Part B: Fundamentals, vol. 64, no. 5, pp. 365-387, 2013.

[34] D. C. Knupp, R. M. Cotta, and C. P. Naveira-Cotta, "Fluid flow and conjugated heat transfer in arbitrarily shaped 
channels via single domain formulation and integral transforms," International Journal of Heat and Mass Transfer, vol. 82, pp. 479-489, 2015.

[35] D. C. Knupp, R. M. Cotta, C. P. Naveira-Cotta, and S. Kakaç, "Transient conjugated heat transfer in microchannels: integral transforms with single domain formulation," International Journal of Thermal Sciences, vol. 88, pp. 248-257, 2015.

[36] A. Beskok and G. Karniadakis, Microflows and Nanoflows: Fundamentals and Simulation. Interdisciplinary Applied Mathematics, Springer, Berlin, Germany, 2005.

[37] A. Adrover, F. Creta, M. Giona, M. Valorani, and V. Vitacolonna, "Natural tangent dynamics with recurrent biorthonormalizations: a geometric computational approach to dynamical systems exhibiting slow manifolds and periodic/ chaotic limit sets," Physica D: Nonlinear Phenomena, vol. 213, no. 2, pp. 121-146, 2006.

[38] F. Garofalo, A. Adrover, S. Cerbelli, and M. Giona, "Spectral characterization of static mixers. the s-shaped micromixer as a case study," AIChE Journal, vol. 56, no. 2, pp. 318-335, 2010.

[39] S. Cerbelli, A. Adrover, F. Garofalo, and M. Giona, "Spectral characterization of mixing properties of annular mhd micromixers," Microfluidics and Nanofluidics, vol. 6, no. 6, pp. 747-761, 2009.

[40] M. Giona, A. Adrover, S. Cerbelli, and F. Garofalo, "Laminar dispersion at high Péclet numbers in finite-length channels: effects of the near-wall velocity profile and connection with the generalized Leveque problem," Physics of Fluids, vol. 21, no. 12, p. 123601, 2009.

[41] S. Yu and T. A. Ameel, "Slip-flow heat transfer in rectangular microchannels," International Journal of Heat and Mass Transfer, vol. 44, no. 22, pp. 4225-4234, 2001.

[42] S. Yu and T. A. Ameel, "Slip flow convection in isoflux rectangular microchannels," Journal of Heat Transfer, vol. 124, pp. $346-355,2001$. 